\title{
THE AESTHETIC AND COMMUNICATIVE VALUES OF ILLUSTRATIONS USED IN INFOGRAPHIC
}

\author{
Amal Farag SOLIMAN *
}

Department of Printed Design, Faculty of Fine Arts, Alexandria University, Egypt

\begin{abstract}
Illustrations are one of the communication tools that have been able to create for themselves the aesthetic and communicative characteristics and features that are unique to them from other communication tools. This field has been able to create for itself an artistic field that has changed and developed throughout history according to changing political and social conditions and technological progress, as well as with the development of various artistic styles. The relationship between drawings and texts was a close relationship that combines imagination, creativity and skill to express specific information or tell stories visually and create new worlds and bodies, so the drawings accompanying the texts acquired many visual and functional characteristics and characteristics in addition to providing us with a visual heritage that objectively reflects our culture. The illustrations have proven their ability to adapt to information design in communicating information and details and how they affect the perception and understanding of the recipient, as they give the viewer a detailed picture of topics that are difficult to see with the naked eye. The illustrations used in the design of the information have visual and aesthetic properties that distinguish them from other typographical elements that can appear in any design.

Keywords

The Aesthetic, Communicative, Values, Illustrations, Infographic.
\end{abstract}

\section{Introduction}

Today's Illustrations are one of the most important means of communication that occupy special importance because of their ability to clarify ideas and meanings, especially those that are difficult to express in words only. This importance is evident in the news cartoons, which are called the term (Infographic or Information Graphics), which appear widely and varied through print media such as newspapers, magazines and books. Words, to clarify lengthy or complex information such as locating or expressing numbers and various indicators, which other communicative elements such as photographs are unable to explain. Information design fees are more stimulating for mental and intellectual capacities, as they can list information with a degree of detail that is impossible to include with any other type of fee.

Opinions have differed as to what precisely defined and descriptive illustration. Any attempt to define an illustration always has many different points of view. For some, drawings are a form of visual communication, or it is an activity to solve communication problems or a means of social and press commentary or explanation. To others, drawings can be applied art in a commercial context or a widespread human narrative art. Finally, some claim that all contemporary arts are in fact now illustrative and descriptive. What makes these drawings widespread and interesting is that they fulfill the imperative of communication, combining

* Corresponding author: finea-dean@alexu.edu.eg 
imagination, creativity, skill, visual narration, and the creation of new worlds and bodies. The drawings vary in purpose as well as in the media and artistic styles. The purpose of the drawings may be to tell a story or clarify a concept or idea.

Among these classifications are the graphics used in the design of the information Infographic, as these drawings serve to clarify the information contained in the text, so these drawings are accompanied by a few words, to clarify the lengthy or complex information such as specifying locations or expressing numbers and various indicators. This type of fee falls within the serious fees that convey a specific reality.

\section{Brief History:}

Perhaps the first images that can be considered "infographics" are, in fact, cave paintings. Although these are not infographics that most people are familiar with today, they still serve a similar purpose of putting a difficult topic, such as a story or lesson, into an easily understandable visual format. Hieroglyphs and maps can be considered to have similar purposes (5).

The term Infographics was first used in 1970 in London graphic design consultancy as one of the branches of graphic design, and as a distinct type of graphic design to display information or other types of design. It was taught as part of graphic design courses. Then the term infographics came to prominence when it was published in the Information Design Journal in 1979. It is used by graphic designers. Since then, the term infographics has increased in effective use. One of the most famous print infographics in newspapers at the time for the British Sunday Times was Peter Sullivan (1932-1996), graphic designer and head of graphic design at Canterbury College of Art. It is common among researchers and professionals alike to believe that infographics, in its modern form, began to take off strongly in the United States of America in 1982. As for generalization and spread, it was with the emergence of easy-touse personal computers, software for graphic processors and software to help in making graphic statistics. .

And the illustrations in the infographic played an important and effective role in clarifying the information. National Geographic is one of the first to provide information design (Infographic) in the way it is now known, that is, the visual design of information in the form of a design that combines images, graphics and texts illustrated by the information). Through this, aspects of history, science, technology, geography, the natural world and human influence on it are depicted.

\section{Infographic Concept:}

What is Infographic? To better understand the definition of the infographic, it is helpful to 
know where the term came from. Infographics are a piece of information and graphic design. Therefore, an infographic is a visual representation of information that aims to make the data easy to understand at first sight. The infographic allows for minimal use of Text can be a powerful tool for presenting data, explaining concepts, simplifying presentations, drawing in detail for different relationships, showing common trends and providing basic insights. The use of images and graphics used in infographics can make an abstract idea more easier to understand and understand. Infographic's popularity in marketing and education) The infographic simplifies large, high-level data sets and makes them easier to understand at first glance.It helps in transferring data in a compact and shareable format.

The aesthetic characteristics and features of the drawings used in the infographic:

The graphics used in Information Graphics are called the term scientific (informative or illustrative) graphics and are used here to refer to the drawings whose function is to clarify, as these drawings serve to clarify the information contained in a subject, and these drawings are accompanied by a few words, to clarify the lengthy information or As complex as identifying sites or expressing different numbers and indicators. Scientific drawings are included in the serious fees that convey a certain reality.

The illustrations in the infographic play an important and effective role in clarifying information that may not be explained by verbal language, the written word, or the photograph, and the illustrations have the abilities to create an illustrative visual field of information.

The scientific drawings used in the infographics can show something on the subject that cannot be photographed or seen with the naked eye, such as detailed information about the process of photosynthesis. Also, scientific drawings are used to improve detail, as these drawings can explain specific details more clearly than photographs. For example, small engine parts that may be difficult to see or photograph can be enlarged, and these parts can be labeled on their descriptions.

Drawings can also remove unwanted and unnecessary parts that may distort the message, which may force the eye to focus on important characteristics and details. Illustrations may also be used to support photographs. A subject about a building fire is what photographs can portray the fire from the outside, but it does not. You can tell us about how the fire broke out from the inside or about the way the firefighting forces used to put out the fire, while the illustrations can cover the deficiencies that the photographs failed to provide.

Information graphics, in general, are more stimulating for mental and intellectual capabilities, because they appeal to all literal and visual areas of the brain. These fees can list information with a degree of detail that is impossible to include with any other type of fee). In this type of 
painting, the artist must be realistic, far from imagination, due to the nature of the subjects associated with this type of painting, which needs to be described and clarified details that already exist, and here lies the importance of the artistic skill of the artist and his vision of the subject away from his own imagination.

In any case, there are two basic tasks for these drawings: gathering information, and finding the appropriate way to express that, as these drawings must present many variables and include and organize them in a way that makes it easier for the reader to obtain the many information it contains in one drawing. In fact, this process is more complex than it appears, but modern technologies and computer programs have made this process relatively easy to produce attractive graphics.

Maps, charts, longitudinal and cross sections of bodies, drawings of particles and their details, and anthropomorphism of mechanical devices, machines and other drawings that illustrate and explain scientific topics in various branches of science: medicine, nature, astronomy, sports, industry and others, all are scientific drawings that require specialized technical hands that draw limited shapes and embody volumes. And its logical dimensions and the detail of its parts with very accurate and clear artistic lines.

Such drawings are considered as a complementary means of illustration to the scientific topic that needs explanation, clarification and support for scientific facts and the delivery of the largest amount of information to the reader. Infographic graphics are used when words alone are unable to convey complex information in a way that is easy to understand. The cartoons also lend serious material such as political, economic and military news a measure of vitality. By combining illustrations, facts and text, infographics often tell a visual story. The infographic may use data visualization to achieve its goal. When designed effectively, infographics provide a self-secret or an overview of a topic, using illustrations to guide the visual stories. Since the infographic conveys multiple ideas, it must be designed in a way that achieves the visual appeal and general understanding of the reader. Because infographics guide the viewer toward conclusions, the design must complement the visual story it is trying to tell.

Reasons to use illustrations in design without other graphic elements:

When a designer has to choose between using illustration, photography, or other graphic elements in the implementation of his design, sometimes this choice is unclear. Both have creative advantages and disadvantages. Differ in costs, time and resources required; Your message can also be communicated in very different ways, whether it is abstract, conceptual, realistic, literal, and in general there are five reasons a designer can guide when choosing illustrations over photography for his next design: 
1- If the designer wants to express something just abstract: Sometimes the nature of the idea or concept he is trying to convey will determine whether the illustration is the best choice. Abstract topics, or topics in which no specific visual elements come to mind, will find it best to resort to illustrative treatment.

2- If the topic is too ambitious: Sometimes, you need your imagination to be the limit, not the budget or practical logistics of what you can portray, where and how. The simple beauty of illustration is that you can depict literally anything you like. Futuristic, surrealistic, or imaginative scenes can be achieved using photography in theory, but can cost a small fortune. If a certain degree of realism is important to achieve, a photomontage or collage can be used to create illustrations. However an artist or designer must choose the appropriate style for illustration, there are no limits to what kind of world an artist or designer can create. But if you are mandating to use a custom type of illustration, you must make sure you are heading in the correct direction that you need.

3- If you are the designer who wants to tell a story: Storytelling may become a ringing word in branding and advertising, but the fact remains that compelling narration can be very effective when it comes to engaging consumers, and acquiring the brand's personality and purpose. Illustration is a great way to take people on a journey, whether you're taking people on a literal tour through the brand story using illustrated scenes or characters, or building a more accurate narrative across the brand's different touch points. Illustrations can be an effective way to represent a brand's position in an easily tangible and reliable way, as well as provide countless options for expansion across various programs. An illustration can also convey specific processes, or key issues important to a brand - its eco-friendly credentials, or educational initiatives, for example - in interesting, engaging, and completely detailed ways.

4- If the designer has some data that he wants to make visually clear: Infographic, in essence, makes complex information easier to absorb and understand by converting it into a visual form. Here the illustration steps stand out in a way that photography can never do: With a touch of creativity, a boring spreadsheet or a list of facts and figures can become a treat for the eyes. On making complex information easier to digest and understand by converting it into visual form. Here the illustration steps are brought into the painting in a way that photography can never do: with a touch of creativity, a boring spreadsheet or a collection of facts and figures can become a treat for the eyes, given the visual aesthetic properties of the illustrations. Whether the illustrations relate to depicting corporate statistics in an annual report, presenting a new perspective on a global phenomenon or illustrating a complex process, the general outlook and characteristics of the infographic are sure to remain. 
5- If the designer is required to fulfill a specific style: The best photographers may have a distinct style, but in the end, the camera never lies. The target will always be the star, and although a carefully guided photography campaign will help strengthen the brand, it will never be as distinctive as a specific style of illustration. Illustration can unify a design and completely transform its look, style, and status - whether it's a multi-program campaign, a series of event graphics or a simple brochure. Here we find that choosing the right method is crucial. As cameras increase in quality, lower prices, and increase in image quality, high-quality photography has become more accessible to brands of all shapes and sizes. Illustration can help distinguish the brand from the crowd in a much more distinctive way, namely carving out a niche defined by the personality and the unique and distinctive attitude.

\section{Infographic Characteristics and Features:}

The basic material for infographics is data, information and knowledge in the form of lines, geometric shapes, arrows, various symbols and illustrations. And we see many of the infographic works that appear in a complex way and with abstract styles and patterns. In order for those meanings contained in the work of the infographic to be properly translated and interpreted, the viewer needs to have visual capabilities, culture and pictorial know-how at a certain level. Even infographic works can be read. Which seeks to create and enhance confidence in the users of the products of this design. The most important characteristics of information graphics can be summarized in the following points:

Visual reduction of a large amount of information. Organize massive amount of data.

- The ability to register.

A way to explore and shed light on hidden and mysterious information.

- Explore new meanings and knowledge through the design scheme.

- Simplifying complex information and making it easy to understand and rely on visual effects to communicate information.

- Converting information and data from boring numbers and letters to interesting pictures and drawings.

- Shortening the time. Instead of reading a huge amount of written data, it can be easily scanned visually.

- The effectiveness of its use in settling through social media to attract more customers and customers for company owners.

- This type of fee is used as a solid code within the code of the website and blog page, which reduces the pressure on the Internet compared to the usual fees and pictures.

- Ease of publishing and spreading the infographics through social networks. 
- Enhancing the ability to think and connect and organize information.

- Helping to retain information a lot of time.

- Applicability to a large number of disciplines and different fields of data (images, numbers, sound).

- The ability to communicate through it and transfer information to others in different languages

\section{Areas of Use of Illustrations in Infographic:}

1- Scientific fees:

Scientific illustrations in both traditional and digital formats provide visual interpretation and aid the viewer by illustrating complex descriptive information. Thus, the function of scientific drawings is to inform, explain and direct briefly and effectively on the viewer in order to communicate information. In this type of graphic, the main focus is on the accuracy of detail in visualizing the subject. The details of this topic must be properly defined to show the proportions, anatomical structures, and other diagnostic features. The artist must have sufficient awareness of the subject matter so that the illustration appears realistically and naturally. There are many artistic techniques that an artist can use in creating scientific illustrations, from traditional techniques such as wood films, watercolors, acrylics, and ink to digital techniques.

The following sections fall under this type of fees:

1- Medical Illustrations:

2- Geology fees:

Geology is the mineralogical science of Earth's solid layers, the rocks that make up them, and the processes through which they change over time. Geology can also include the study of the solid features of any planet, such as the geology of the Moon and Mars). Geology gives insight into the history of the Earth by providing initial evidence of the Earth's mass, the history of the evolution of life on it, and past climates. Geology is concerned with exploring and exploiting minerals and hydrocarbons, assessing water resources, understanding natural hazards, addressing environmental problems, and providing insight into past climate change. The illustrations analyze these phenomena through studying and depicting them in a visual form and explaining them in detail, such as analyzing the elements of rock formation.

3- Technical Illustration:

Technical drawings are one of the types of graphics that are used to visually communicate with information of a technical nature, to illustrate structural structures that require specific dimensions with different dimensions, dimensions, sectors, and different viewing angles.

4- Fees for fictional topics: 
They are drawings that are concerned with historical, religious or fictional topics .... through the different places, characters and events that need explanation.

Technical methods and techniques used to implement illustrations in information design:

The artistic style is the set of laws or the visual code that reflects or summons the aesthetic feeling that may suggest and express the content of the story and the general atmosphere of it). And it is the artistic style that determines the place of the artist from the time. It is he who distinguishes between the old and the modern, the modern from the immortal, the real from the false. And style is a major concern for an artist at any stage of his career. The point of view of the artist and how to express his ideas is what achieves success or failure for the artist, as it may mean that the artist has a clear voice or without a voice. When looking at the artistic style as the voice of the artist, this does not mean that it is possible to turn it on or off as desired, but rather it is an issue that is endemic to the personality of the artist.

The technical methods that are used to implement illustrations in designing information vary according to the nature of the topic and the medium in which the infographic will be published, if it is printed such as magazines or books, or digitally such as websites.

1- Realistic literal representation: the drawings executed in this manner are distinguished by the real and direct embodiment of all the expressive details represented by the artist's eye to be recorded in the illustration. And here we are dealing with mass-produced cartoons to convey reality without exaggeration. In this sense, the drawings here are a representation of a subject, whether large or small, with the necessary components and elements such as people, beings and other things, in a situation or interacting together in a manner that is visually credible. And there will be a certain amount of interest in achieving precision with regard to the elements and placing them in perspective and proportions.

This is because the silhouettes are more realistic and perhaps more representative, because the drawings - whatever the mediums used in their implementation are traditional or even digital are achieved by the shadow effect with tonal degrees to define all aspects of the image and thus appear realistically.

2- Analytical representation: This method is used to study and analyze the shape, composition, proportions, geometric structure, area, and the relationship of the elements that are studied individually or in groups.

3- Iconographic representation: the information is expressed in a pictorial symbolic form in an abstract manner.

4- Geometric representation: This method is used with topics of a technical nature.

This type is concerned with the transfer of information and statistical data based on numbers 
and numbers, and their representation in a graphic form.

\section{Results:}

1- Illustrations are a powerful communication tool with distinct visual properties from other communication tools.

2- The great importance of illustrations in print and digital media since their inception and their use as a primary means of communicating information to readers due to their visual and communication functions.

3- The methods of illustrations used in designing the information vary according to the topic presented in the infographic.

4- There are many media and techniques that can be used to implement the illustrations included in the infographic.

\section{Recommendations:}

1- The designer must ensure the integration of the typographical elements (color, text shape, size, spaces ...) with the illustrations in the infographic to achieve the purpose of all these different visual elements. Choosing carefully which of these elements will fit the visual message.

2- The researcher believes that it is necessary to take advantage of technological development and the digital revolution, both in terms of designing information and implementing illustrations.

3- The researcher also recommends the necessity of using illustrations for their optimal use in designing information in line with its visual and communicative characteristics.

\section{Reference:}

1) Fahd bin Abdul Aziz Badr Al-Askar: Press Production (its functional importance and its modern trends), Al-Obeikan Library, Riyadh, 1998.

2) Abdulaziz Saeed Al-Suwai'i: Press Production and Design (Between Ideas, Films and Computers), Dar Al-Multaqa for Printing and Publishing, Lebanon.

3) Mark Wigan: Basics Illustration (Text and Image), AVA Publishing SA, Switzerland, 2008.

4) John Morrish: Magazine Editing, Rutledge, USA, 1996.

5) Mark Simiciklas, The power of Infographics, Que publishers, Indianapolis, United States of America, 2012.

6) Jennifer George: Practical guide graphics reporting, Elsevier Inc., Oxford, 2006.

7) Lawerence Zeegen: The Fundamentals of Illustration, AVA Puplishing SA, 2005.

8) Amy E. Arntson: Graphic design basics, Thomson Wadsworth, USA, 2007.

9) Jennifer George: Practical guide graphics reporting, Elsevier Inc., Oxford, 2006. (8)Laura 
Mol: The Potential role of Infographics, Master Thesis, Amsterdam University, 2011.

10) Andrew Loomis, Creative Illustration, The Viking Press, New York, 1947.

11) Alan Male: Illustration (A Theoretical \& Contextual Perspective), AVA Publishing SA, Switzerland, 2007.

12) Salah Shaaban Hassanin ABDEL RAHMAN, FORGING ON COPPER BETWEEN CRAFT AND CREATIVITY, International Journal of Multidisciplinary Studies in Art and Technology, Vol. 2, No. 1, 2019, pp. 1-8.

13) Shaima Salama Ibrahim DESOUKI, FORMULATIONS FOR GLASS PAINT USING

LOCAL PAINTS, International Journal of Multidisciplinary Studies in Art and Technology, Vol. 2, No. 1, 2019, pp. 9-11.

14) https://icons8.com/articles/what-is-an-infographic/ 14-2-2019

15) https://visme.co/blog/what-is-an-infographic $114-2-2019$

16) https://killerinfographics.com/blog/data-visualization-versus-infographics.htmL

17) https://en.wikipedia.org/wiki/Infographic

18) https://www.creativebloq.com/advice/5-reasons-to-use-illustration-in-your-designs

19) http://www.jenunderwood.com/2015/11/23/storytelling-infographics-start/

20) http://markrhugraphics.blogspot.com.eg/2014/03/storyboard.html

21) http://paulweston.info/geological-illustration

22) https://www.behance.net/gallery/51079233/3D-Infographics-andTechnical-Illustrations

Received: September 15, 2019

Accepted: November 20, 2019 